\title{
Un lugar para la historia y la filosofía en la enseñanza de las ciencias
}

\section{Michael R. Matthews}

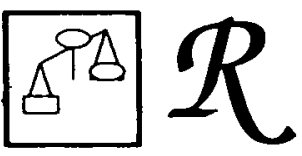

A partir de las críticas al método clásico de enseñanza de las ciencias asi como a la alternativa que trató de plantear la enseñanza «por interrogación», el autor analiza las promesas y el papel actual de un enfoque bistórico y constructivista en la enseñanza de las ciencias.

Han pasado treinta años desde las últimas grandes reformas en educación científica. Muchos creen que es tiempo de revisión de aquellos primeros cursos y de renovación de la educación científica, de sus contenidos, propósitos, métodos. También, y es importante, hay un interés renovado en la preparación de profesores de ciencias. Crecen en número quienes creen que la Historia y la Filosofía de la ciencia tienen un lugar importante en esta renovación. Nadie niega que ha habido demasiada poca interrelación y cooperación entre filósofos e historiadores de la ciencia por un lado y educadores científicos por otro. $\mathrm{Y}$ no es bueno, porque en todo nivel donde ha habido cooperación, la educación científica se ha beneficiado sensiblemente, sea en el desarrollo de cursos, la producción de material de enseñanza, la redacción de libros de texto, la dirección de la investigación sobre el aprendizaje de las ciencias, la ampliación de clases y actividades, la preparación de profesores o la definición de competencia científica. El presente proyecto internacional descrito por mí en la introducción a este volumen contribuirá humildemente al avance de la interrelación y cooperación. Dada la huida de las clases de ciencias que ha tenido lugar particularmente en América, se impone una acción conjunta para revisar y mejorar la situación.

Las reformas de finales de los 50 y principios de los 60 tenían ciertos objetivos, siendo el más importante la creación de «pequeños científicos» (Roberts, 1982). Aprender sobre la ciencia, su historia, Filosofía y contexto social eran preocupaciones menores. En los Estados Unidos, el Sputnik ruso inspiró el Proyecto de Ley de Educación para la Defensa Nacional (National Defense Education Bill) de 1958 que puso en acción unos cuantos cursos nuevos de ciencias (Elbers \& Duncan, 1959). Se los conoce por sus siglas, PSSC, CHEM, CBA, BSCS y ESCP. La Fundación Nacional de las Ciencias (National Science Foun- 
dation) apoyó más de veinte nuevos cursos de matemáticas y ciencias, y para 1968 tres millones y medio de estudiantes participaban en ellos (Duschl, 1985 p. 547). Fueron diseñados en general por científicos académicos pensando en el alumno brillante interesado por las ciencias. El informe Rockefeller de 1958 sobre la búsqueda de sobresalientes en la educación americana representa las esperanzas de la época. Al mismo tiempo, los planes de Nuffield se introducían en Inglaterra y los cursos de Messel y ASEP en Australia. Ni la Historia ni la filosofía de la ciencia tenían mucho sitio en estos cursos, aunque ciertamente asumían la visión empirista de estas materias entonces en boga. Naturalmente esto quedaba aún más claro en los libros de texto que acompañaban a los cursos.

En la mayoría de los cursos el método de enseñanza recomendado era el «método interrogativo» inspirado en Bruner. Se reconocía que los antiguos métodos de instrucción eran poco eficaces y contraproducentes. Pocos alumnos aprendían la asignatura y muchos abandonaban las ciencias a la primera oportunidad que se les presentaba. Una situación comparable a la del cambio de siglo cuando uno de los primeros reformadores, Henry E. Armstrong, observaba,

«No tengo duda en afirmar que en el día de hoy eso que llaman ciencia y que se enseña en casi todas las escuelas, especialmente la que piden los examinadores, no sólo no tiene valor, sino que es realmente perjudicial.» (1903, p. 37)

Opiniones similiares había expresado Ernst Mach, prominente físico y filósofo, que tal vez fuera el primer científico importante que se interesó por elementos de pedagogía científica. Mach había dicho

«No conozco nada más terrible que las pobres criaturas que han aprendido demasiado. Lo que han adquirido es una telaraña de pensamientos demasiado débil para sostener postulados sólidos, pero con la complicación suficiente para producir confusión». (Mach, 1895, p. 367)

En contra de los antiguos métodos didácticos, los nuevos cursos insistían en aprender actuando, o en la ciencia como interrogación. Un crítico, hablando de las reformas, describía su optimismo

«El curso de Física PSSC, como muchos cursos posteriores auspiciados por la NSF (National Science Foundation (Fundación Nacional de las Ciencias) se pensó de tal modo que el estudiante aprendía haciendo experimentos, efectuando sus propias observaciones y generalizando a partir de su experiencia de primera mano». (Crane, 1986, p. 65)

Joseph Schwab, el educador de ciencias que tanto ha publicado, postulaba «la ciencia como interrogación» y defendía la racionalidad de los nuevos cursos (Schwab, 1962).

Con el paso de treinta años y el beneficio de ser capaces de mirar atrás, pueden hacerse y se han hecho juicios sobre las reformas. El modelo inductivo de interrogación en que se basaban probó ser deficiente o al menos altamente controvertido incluso en el momento en que los cursos se implantaban. Pronto las críticas de Kuhn, Popper, Toulmin, Laudan y otros volvieron insostenibles algunos de los supuestos cruciales del programa de ciencias como interrogación. Los programas de la NSF y Nuffield suponían que el estudiante llegaba a las ciencias como una «tabula rasa». Tales suposiciones no deberían hacerse. Los alumnos tienen a menudo creencias y conceptos intuitivos profundamente arraigados sobre los procesos naturales, y estos afectan al modo en que se ve el mundo, cómo se entienden los nuevos conceptos y cómo «pregunta» el alumno. Se ha investigado mucho en la dirección de la idea piagetiana de que estas creen- 
cias intuitivas reflejan estadios anteriores de la Historia de la ciencia. Por las razones que sean, está claro que las reformas de los 60 han agotado su curso. Tanto alumnos como profesores abandonan la clase de ciencias. Un artículo crítico representativo sobre las reformas ha dicho

«A pesar de los nuevos cursos, los profesores mejor preparados y la mejora de instalaciones y equipos, las expectativas optimistas de que los estudiantes se hicieran preguntas se han alcanzado muy pocas veces». (Welch et al., 1981, p. 33)

\section{El mismo artículo seguía diciendo:}

«la segunda mayor reserva que expresaban los profesores sobre la enseñanza según el método interrogativo era que no servía para la mayoría de los alumnos. Lo ven como causa de confusión y demasiado difícil excepto para el alumno más brillante».

Está claro que los cursos y los procesos de enseñanza habrían salido beneficiados si los filósofos y los historiadores hubieran participado más en la tarea reformadora desde el principio.

Algunos de los problemas que provocaban los nuevos cursos se identificaron pronto. La Asociación británica para la Educación Científica (Assocition for Science Education, ASE) en su Training of Graduate Science Teachers (Preparación de Profesores Licenciados en Ciencias) señaló el problema obvio de profesores que no comprendían o no tenían interés en la naturaleza de la propia ciencia. Decía de los profesores

«Muchos actúan y piensan científicamente como resultado de su preparación, pero carecen del entendimiento de la naturaleza básica y propósitos de la ciencia». (Association for Science Education, 1963, p. 13)

La misma entidad, en su informe de 1979, Alternatives for Science Education, (Educación Científica Alternativa), exponía que era deseable una educación científica para todos los estudiantes hasta los dieciséis años. Tal programa «incorporaría un equilibrio razonable entre los aspectos especializados y generales de la ciencia y debería reflejar» la ciencia como una «actividad cultural». En un informe posterior, Education Through Science (Educación a través de la Ciencia, 1980) la enseñanza de la ciencia como una actividad cultural fue descrita como

«la búsqueda más generalizada de la cultura y el conocimiento científicos que toma nota de las implicaciones históricas, filosóficas y sociales de las actividades científicas y, por lo tanto, lleva a un entendimiento de la contribución que la ciencia y la tecnología hacen a la sociedad y al mundo de las ideas».

La ASE ha reconocido explícitamente la importancia de la Historia y la Filosofía de la ciencia en su propio proyecto Science in Society (Ciencia en la Sociedad), que incluye un lector de la materia, (Ramage, 1983).

Manuel, en un comentario sobre el sitio de la Filosofía en la enseñanza científica británica, reconocía el declive de la competencia científica en la escuela y postulaba la inclusión de programas de Historia y Filosofía en cursos de preparación para profesores.

«Esta base más filosófica que se propone para los profesores los capacitaría, según se cree, para llevar a cabo su enseñanza científica de un modo más informado y versátil y de estar en posición más efectiva para llevar a sus alumnos a construir un cuadro coherente de la ciencia, adecuado a edades y capacidades, que tantas veces se echa de menos» (Manuel, 1981, p. 771). 
Este proyecto hace la misma llamada.

En los EE.UU. se huye de la ciencia de forma dramática. En 1985-86, 7.100 Institutos no tenían cursos de física, había 4.200 sin química y 1.300 sin biología. Entre 1971 y 1980 hubo una caída del $64 \%$ en el número de pre-licenciados en cursos de enseñanza de ciencias. Se estima que el $30 \%$ de los profesores de ciencias no están cualificados para enseñar la materia. Treinta y cinco estados permiten graduarse en el Instituto con poco o nulo estudio de ciencias, hecho reflejado en una investigación reciente a nivel nacional que descubrió que el $50 \%$ de los jóvenes de diecisiete años era incapaz de hallar el área de un cuadrado dada la longitud de un lado. Todo esto aparece documentado en muchos informes publicados, particularmente, $A$ Nation at Risk (Una nación en peligro, Bybee et al., 1984; Dowling \& Yager, 1983), National Comission on Excellence in Education (Comisión Nacional de Sobresalientes en la Educación, 1983; Weiss, 1988). No es sorprendente que en los Estados Unidos haya tantas llamadas a la concertación de nuevos exámenes y a la reforma de la educación científica. (Bybee 1977; Bybee et al., 1984; Gallagher y Yager, 1981; Hurd, 1984; National Science Board, (Junta Nacional de las Ciencias, 1983), Yager y Penick, 1985).

Un indicador de la preocupación americana sobre el estado de la enseñanza de las ciencias en la escuela es el programa nacional de examen a profesores de ciencias, con base en Stanford y fundado por Carnegie. Otros indicadores son las muy debatidas propuestas del Grupo Holmes para la reorganización de la preparación de profesores en las dos conferencias de la Phillips Exeter Academy sobre educación científica y tecnología para el mundo del mañana. Muchas de estas preocupaciones se expresan en el informe de 1983, de la Junta Nacional Científica, Educating Americans for the 21st. Century (Educar Americanos para el Siglo XXI).

En Inglaterra, la proposición gubernamental de un curso científico nacional ha dado urgencia a la pregunta de qué es una educación científica apropiada (Departamento de Educación y Ciencia (Department of Education and Science), 1985; Thompson, 1987). La Asociación para la Educación científica (Association for Science Education, ASE) ha preparado muchos informes llamando urgentemente a la reforma y a la sustitución de los aún ampliamente utilizados programas escolares Nuffield. En apoyo de estos objetivos, la ASE ha fundado dos nuevos proyectos académicos: el curso Science and Society (Ciencia y Sociedad) de 1981 y el curso Science in its Social Context (Ciencia en su Contexto Social), SISCON, de 1983. En contra del acento positivista anterior, la Junta Nacional para la Revisión de Programas Científicos de Enseñanza Secundaria, (National Secondary Science Curriculum Review) dijo en 1983 que los estudiantes deberían obtener «algún conocimiento del desarrollo histórico de las teorías y principios científicos». En 1989, tres cuerpos de examen introducirán un curso superior sobre Historia y Filosofía de la ciencia. Estos desarrollos están aún alejados de la ciencia escolar. Sin profesores que tengan interés y competencia en Historia, Filosofía y estudios sociales de la ciencia y sin un esfuerzo conjunto para convencer a los administradores y legisladores del beneficio que reporta, tales estudios permanecerán alejados.

Los filósofos y los historiadores de la ciencia tienen un papel valioso que jugar en la reforma de la educación científica. Su coloboración con los educadores científicos ha sido demasiado poco frecuente. 


\section{LA HISTORIA DE LA CIENCIA Y EL CURRICULUM}

Los historiadores de la ciencia han mostrado mayor actividad que los filósofos en empresas educativas. Las asociaciones británica y americana de la Historia de la ciencia tienen sus comités de educación. La Asociación Británica fue fundada en 1946 y empezó inmediatamente a colaborar con las entonces Asociación de Maestros de Ciencias (Secience Masters Association) y la Asociación de Profesoras de Ciencias, antecedentes de la ASE sobre cómo podría incorporarse mejor la Historia de la ciencia a los cursos escolares. Mucho antes, en 1971, la Asociación Británica para el Avance de la Ciencia (British Association for the Advancement of Science, BAAS) había pedido con urgencia el acercamiento histórico de la enseñanza de las ciencias diciendo que la Historia de la ciencia era «un disolvente de las barreras artificiales entre los estudios literarios y científicos que establecía el horario escolar» $(1917$, p. 140). Al año siguiente, el Informe J. J. Thompson apoyaba estas afirmaciones (Waring, 1979).

La Sociedad Americana de Historia de la Ciencia (American History of Science Society) participa con la Comisión Nacional de Estudios Sociales (National Comission on Social Studies) y la Asociación Americana para el Avance de la Ciencia (American Association for the Advancement of Science) en proyectos de reforma de programas. Esta última organización patrocina un proyecto de Educación Liberal y el proyecto Ciencias. Casi todo este esfuerzo se dirige al programa de Historia en un intento de que incremente sus contenidos sobre los desarrollos científicos y tecnológicos.

En Europa, al final del siglo xIx, Ernst Mach proponía un acercamiento filosófico e histórico a la enseñanza de las ciencias en la escuela. Fue en su conferencia para maestros de escuela «On the instructions in the Classics and the Sciences» (sobre la instrucción en los clásicos y las ciencias, Mach, 1985). Al mismo tiempo, Pierre Duhem proponía con urgencia el método histórico en la enseñanza de la Física. Fue él tal vez el primero en exponer la analogía entre el desarrollo de la ciencia y el crecimiento de la comprensión individual (una visión desarrollada más tarde por Piaget en su psicología genética). Duhem era sensible a los problemas de la enseñanza científica. Decía que la tensión entre la lógica de la materia y la psicología del alumno «hace de la enseñanza de esta ciencia algo particularmente delicado» (Duhem, 1954, p. 258). Recomendaba que

«El método legítimo, seguro y provechoso, de preparar al alumno para recibir una hipótesis de Física es el método histórico. Remontarse a las transformaciones que sufría la materia empírica mientras la forma teórica se apuntaba por primera vez. Describir la larga colaboración por medio de la cual el sentido común y la lógica deductiva analizaban la materia y modelaban aquella forma hasta que una se adaptaba exactamente a la otra. Es la mejor manera, seguramente la única, de dar a quienes estudian Física una visión correcta y clara de la organización real y altamente compleja de esta ciencia». (Duhem, 1954, p. 268)

Se ha desarrollado un número pequeño de importantes programas científicos bien documentados históricamente. En América, esta colaboración entre historiadores y educadores ha sido particularmente productiva en la Universidad de Harvard. James Bryant Conant, presidente de la universidad y físico e historiador, escribió libros muy difundidos postulando un acercamiento histórico a la pedagogía científica. También preparó el influyente Case Studies in Experimental Sciences (Estudios de casos de ciencia experimental, Conant, 1964). Tho- 
mas Kuhn, el filósofo de la ciencia más ampliamente leído en las últimas tres décadas, ha dicho que Conant «Me convenció el primero de que el estudio histórico podría producir un nuevo tipo de entendimiento de la estructura y función de la investigación científica». (Kuhn, 1977, p. xi) Otro físico de Harvard que entendía su disciplina en términos históricos era Gerald Holton, que a principios de los 60 colaboró con educadores científicos para producir el Harvard Proyect Physics Course (Curso de Física Proyecto Harvard) (Ver Holton, 1978). Holton era miembro de la Comisión Nacional de Sobresalientes en Educación que produjo Una Nación en Peligro y ha comentado lo que ha supuesto para la educación científica (Holton, 1986).

La Física del Proyecto Harvard ha sido un ejemplo de cómo la Historia de la Ciencia puede contribuir al desarrollo de cursos científicos. El éxito del curso es prometedor para las propuestas académicas defendidas aquí. Del mismo modo hay que aprender la lección que nos dan sus deficiencias (Brush, 1987; Holton, 1988; Russell, 1981). Fue el único de los nuevos cursos que consideraba seriamente la Historia y el contexto cultural de la ciencia. A mediados de los 70 el $15 \%$ de los alumnos de cursos de Física en América estaba en el curso de Física del Proyecto. Estos alumnos aprendían a pensar no en términos de uns «método científco» estereotipado, sino que valoraban los papeles que jugaban diversos acercamientos, imaginación, confirmación e instrumentación, en la búsqueda del conocimiento científico (Aikenhead, 1974). Y su conocimiento de la disciplina no sufrió por ello (Brush, 1987, p. 78).

Un asunto reseñado en los artículos críticos sobre la Física del Proyecto es el papel importante del profesor en la efectividad del programa. Wayne Welch afirma que casi todos los informes de investigación indican que no más del $5 \%$ de la desviación del aprendizaje en el alumno se debe al programa. El resto se debe al profesor (Welch, 1979). Por esta razón los americanos intentan desarrollar programas «a prueba de profesores». Pero otra solución es asegurarse de que los profesores tienen buenos fundamentos de Historia y Filosofía de la ciencia y que su propio entusiasmo y competenca hagan el resto.

El Estudio Programado de Ciencias Biológicas (Biological Science Curriculum Study, BSCS), introducido a principios de los $60 \mathrm{y}$ adaptado a muchos países incluyendo Israel y Australia, era un ejemplo de programa con amplia documentación histórica. En su desarrollo colaboró Josephy Schwab. Ciencia como interrogación era su lema. Para Schwab

«La esencia de la enseñanza de la ciencia como interrogación sería mostrar algunas de la conclusiones de la ciencia en el marco de la forma en que surgen y son compro. badas» (Schwab, 1962, p. 40).

El beneficio de un acercamiento histórico a la enseñanza escolar de la cien. cia ha sido bien examinado. Se alude sobre todo a su capacidad de servir de puente entre las «dos culturas», retratadas dramáticamente por C. P. Snow. La investigación sobre cursos, rendimiento escolar y actitudes ha establecido otros resultados positivos, incluyendo una comprensión creciente de la ciencia, un interés creciente en la materia, un pensamiento crítico potenciado y la participación reciente de las chicas en programas científicos escolares (Brush, 1974-1987; Finocchiaro et al., 1980 Klopfer, 1969; Kauffman, 1979; Russell, 1981; Sherratt, 1982). La Historia y la Filosofía dan rostro humano a la ciencia y de algún modo superan su imagen cienticifista (Brush, 1978; Omerod, 1971).

En tono discordante, Thomas Kuhn defendía que la enseñanza adecuada de la historia de la ciencia a los niños en la escuela corroía el aprendizaje cientí- 
fico «normal». Opinaba que en una clase de ciencias, la Historia de la ciencia debería falsearse, que los primeros científicos deberían representarse como si hubieran trabajado sobre «la misma serie de problemas fijos» que los científicos modernos (Kuhn, 1970, p. 138). Dado que el contenido de la ciencia es complejo, no parece haber mucho motivo para que la Historia de la ciencia en clase no pueda ser compleja a su vez. Karl Popper decía del «científico tipo» de Kuhn, que se le había enseñado mal, que tenía técnica, pero no comprensión.

Vale la pena señalar que el argumento principal para introducir la Historia de la ciencia en la educación científica es que favorece un mejor aprendizaje científico. Es el punto que repetía Ernst Mach hace un siglo. Decía entonces que

«Quien conozca sólo un punto de vista o una forma de ver las cosas no cree que otro haya estado nunca en su lugar ni que otro venga después; ni duda ni hace pruebas». (Mach, 1911, p. 17)

\section{HISTORIA DE LA CIENCIA Y PSICOLOGIA DEL APRENDIZAJE}

Jean Piaget, siguiendo a Duhem, formuló la noción de que en el desarrollo cognitivo la ontogenia recapitula la filogenia.

«La hipótesis fundamental de la epistemología genética es que existe un paralelismo entre el progreso realizado en la organización lógica y racional del conocimiento, (Historia de la Ciencia) y los correspondientes procesos pscológicos formativos, (desarrollo individual)». (Piaget, 1970, p. 13).

Esta afirmación de Piaget ha sido la base de un influyente programa de investigación. Thomas Kuhn decía de Piaget que «parte de lo que se sabe sobre cómo hacer las preguntas que se hicieron científicos muertos se ha descubierto examinando las preguntas de los niños vivos de Piaget». (Kuhn, 1977, p. 21). Y, a la inversa, el historiador de la ciencia Alexandre Koyre observó que fue la física de Aristóteles la que le enseñó a comprender a los niños de Piaget. Este lado de la afirmación de Piaget tiene gran importancia para la enseñanza de las ciencias. El pasado de la ciencia puede iluminar el presente del aprendizaje científico.

Se han elaborado opiniones similares por parte de filósofos de la educación tales como Richard Gregory y Percy Nunn. Bajo su influencia hubo un interés sostenido en la Historia de la ciencia hacia la enseñanza científica secundaria británica en los años 20 y 30 (Brock, 1987). F. W. Sanderson, director de la escuela Oundle de 1892 a 1922, defendía la lectura de fuentes primarias y el retrato de la ciencia como parte integral del desarrollo de la cultura. Su consejo a los estudiantes era

«Leed a Arquímedes... leed los artículos de Faraday... seguid los largos procesos de los experimentos, la diversidad de métodos, los intentos y fracasos, incertidumbres, dudas y provocación, el ambiente del descubrimiento». (Sherratt, 1982, p. 232)

La historia confusa del aprendizaje del descubrimiento en la educación científica puede iluminar la afirmación piagetiana (Atkinson y Delamnont, 1977; Hodson, 1987). Los planes Nuffield, por ejemplo, insistían en que los niños se convirtieran en «científicos por un día». Lo que esto suponía en realidad era algo en principio libre de problemas, los profesores debían guiar a los niños en sus descubrimientos científicos. Es corriente entre profesores decir que cuando se dirige a los niños para descubrir alguna conceptualización, relación o ley sobre los fenómenos que se les presenta, a menudo se les ocurren ideas que son 
sorprendentes como aquéllas que habían sido dominantes en distintas épocas en la Historia de la ciencia real. Este efecto de recapitulación ha sido ampliamente investigado.

Rosalind Driver ha descubierto que los niños tienen una idea orgánica de la combustión, que ven la quema como una liberación de algo flamígero que deja una ceniza (Driver et al., 1985, p. 158). Bartov ha mostrado que las concepciones intuitivas de los procesos biológicos son altamente teleológicas. Como podría esperarse, la comprensión inicial de la evolución es muy lamarckiana (Brumby, 1979; Lucas, 1971). Hay una extensa literatura que muestra la naturaleza fundamentalmente aristotélica de las ideas intuitivas en mecánica (Champagne et al., 1980; Disessa, 1982; McCloskey, 1983; Whitaker, 1983). Se han demostrado estas relaciones por todo el espectro científico. Han sido el tema de docenas de artículos en dos conferencias internacionales, concepciones Erróneas en el Aprendizaje Científico, «Misconceptions in Science Learning» (Ideas Falsas en el Aprendizaje de las Ciencias) (Elm y Novak, 1983; Novak, 1987).

La Historia de la ciencia, la teoría del aprendizaje y la pedagogía se dan cita en la clase de ciencias. Cómo entienden los niños intuitivamente los procesos afectará a la asimilación de ideas nuevas. Este es un punto más bien simple, y fue concretado en las teorías del aprendizaje de David Ausubel y Jerome Bruner. Ausubel, en su trabajo más importante, Educational Psicology a cognitive view (Psicología Educacional, una Visión Cognitiva) estableció que

«Si tuviera que reducir toda la psicología educativa a un solo principio sería éste; el factor simple más importante que influencia el aprendizaje es lo que el estudiante ya sabe. Descúbrase qué es y enséñesele en consecuencia».

Comprender los obstáculos en el desarrollo de la Historia de la ciencia puede en alguna medida arrojar luz sobre problemas del aprendizaje individual. $\mathrm{Na}$ die sugiere una identidad de aprendizajes individual e histórico o que la física aristotélica o la química del flogisto sea juego de niños. Lo que se sugiere es que la Historia de la ciencia nos permite entender mejor cuáles son las ideas actuales y que el conocimiento de los «obstáculos epistemológicos» en el desarrollo de la ciencia puede iluminar problemas similares del aprendizaje individual. Apreciar dónde tuvieron dificultad las grandes mentes orienta a un profesor sobre dónde podrían tener también dificultades mentes menores. Hogg, en un texto de química basada en la Historia ahora anticuado, lo expresaba bien:

«Es desarrollo histórico es un acercamiento lógico. El lento progreso de los primeros siglos se debía a la falta de conocimiento, técnica pobre y ataques sin método. Pero esas son precisamente las dificultades de quien se inicia en la química. Hay un lazo de comprensión entre el principiante y el pionero» (Hogg, 1983, p. VII).

Existe, por supuesto, el peligro de que se enseñe una Historia simplista, aunque con una preparación adecuada de los profesores, no debería preocupar más que si se enseñara mecánica simplista. El peligro contrario es dejar que lo mejor ponga trabas a lo bueno. Está claro que no hay una única «Historia de la ciencia», y es un error fingir que la hay. Las divisiones entre internalistas y externalistas, racionalistas e irracionalistas, permanecen más bien claras. La importancia relativa dada al experimento y a la razón en la mecánica de Galileo, por ejemplo, sigue debatiéndose, pero esto no debería ser causa de preocupación. Los profesores enseñan mejor cuando no llegan con respuestas sino con preguntas que atrapan y estimulan al alumno. Una Historia bien enseñada puede hacerlo. Âlbert Einstein señaló que fue la lectura del trabajo histórico de Ernst Mach, 
The Science of Mechanics (La ciencia de la mecánica) (1983) la que le liberó la mente para contemplar nuevos marcos interpretativos en Mecánica. De su propia educación Einstein dijo,

«Tras aprobar el examen final y durante un año entero me producía desagrado la consideración de cualquier problema científico. Es más, es casi un milagro que los métodos modernos de instrucción no hayan estrangulado la sagrada curiosidad de hacerse preguntas». (Schillp, 1951, p. 17)

Esta dimensión histórica puede ayudarnos a comprender la fortaleza de las creencias intuitivas. McCloskey descubrió que el $93 \%$ de los estudiantes de Física de Instituto a punto de hacer una carrera de Física creían que un objeto adquiría una cierta cualidad de ímpetu cuando se le ponía en movimiento, y que este «ímpetu» mantenía el movimiento. Sorprende poco. También descubrió que el $80 \%$ ¡seguía creyéndolo después de aprobar la carrera! Señalaba,

«Esta idea falsa parece estar fundada en una teoría intuitiva y sistemática del movimiento que es inconsistente con los principios fundamentales de la mecánica newtoniana. La teoría intuitiva guarda un parecedio sorprendente con la teoría pre-newtoniana del ímpetu» (McCloskey. 1983, p. 122).

Muchos otros han señalado esta incapacidad de la enseñanza científica de afectar a creeencias profundamente arraigadas (Archenhold et al., 1980; Brumby, 1989; Champagne et al., 1980; Gilbert et al., 1982; Viennot, 1989). Los alumnos viven a menudo en dos mundos, uno para el examen de ciencias, otro para la vida cotidiana. Esto queda ilustrado alarmantemente con encuestas americanas recientes que muestran que las creencias en la astrología se ven muy poco afectadas por la obtención de un título americano de ciencias. La Historia de la ciencia nos recuerda la dificultad para aprender y asimilar la más mínima de las nociones. Pierre Duhem, al advertir contra el peligro de basar la instrucción científica en el sentido común, había observado a principio de siglo,

«¿Está claro a la luz del sentido común simplemente que un cuerpo en ausencia de fuerzas actuando sobre él se mueve de modo perpetuo en línea recta a velocidad constante? ¿O què un cuerpo bajo un peso constante acelera constantemente la velocidad de su caída? Al contrario, tales opiniones están notablemente lejos del conocimiento que da el sentido común. Su nacimiento se ha debido a los esfuerzos acumulados de todos los genios que se han ocupado de la dinámica durante dos mil años» (Duhem, 1954, p. 233).

El auge del constructivismo en la psicología educativa ha llevado a una mayor apreciación del lugar de la Historia y de la Filosofía de la ciencia en la teoría y práctica de la educación científica. El contructivismo es una variante inspirada en Piaget de la teoría del aprendizaje cognitivo. Joseph Novak en educación científica y Ernst von Glasersfeld en educación matemática son dos exponentes representativos de la postura (Novak, 1987; Glasersfeld, 1987). Es una teoría del aprendizaje anti-empirista anticonductista. Se apoya principalmente en los teóricos de un «marco» en la filosofía de la ciencia -Kuhn, Toulmin, Bachelard-. Con esto, no es sorprendente que los elementos epistemológicos sean corrientes en el constructivismo. La importancia reconocida de la epistemología en el constructivismo se puede ver en la introducción a la gran bibliografía de investigación de ese campo. Ahí, Duit dice

«El término marco alternativo que aparece en el título de la bibliografía representa un programa aceptado por la abrumadora mayoría de los investigadores de nuestro campo. Es un programa que contempla las ideas de los alumnos como ideas de pleno 
derecho y no como ideas falsas que deben ser borradas lo más rápido posible» (Pfundt y Duit, 1988, p. xxxv).

Esto sigue a la introducción de la expresión «marcos alternativos del alumno» en la crítica 1978 de Driver y Easley.

Es fácil ver que el constructivismo depende de posturas de la Historia y Filosofía de la ciencia y que tales posturas pueden contribuir a la exposición crítica del programa. Temas como verdad, realismo y racionalidad se ponen de manifiesto en el debate constructivista. ¿Cómo puede el conocimiento del proceso de cambio de conceptos en la Historia de la ciencia aportar información a los procesos de cambio de conceptos individual y viceversa? Y más aún, ¿qué aspectos epistemológicos suponen ambos? Karl Popper distingue claramente entre el segundo mundo, hechos experienciales, y tercer mundo, teorías científicas. Esta es su «ciencia sin: un sujeto que conoce». El contructivismo levanta un puente entre estos dos mundos, pero el puente requiere apoyo epistemológico.

\section{FILOSOFIA Y ENSEÑANZA CIENTIFICA}

Los efectos de la comprensión psicológica o la falta de ella se ven por todas partes en la educación de las ciencias. Desde afirmaciones de supuesto método científico a relaciones de leyes científicas o bien opiniones sobre la estructura de la ciencia. Sin embargo, los filósofos sólo se han ocupado en raras ocasiones de asuntos de educación de las ciencias. Algo que lamentan Robinson (1969), Ennis (1979) y Martin (1974) en sus importantes críticas. Esta despreocupación contrasta marcadamente con los estudios crecientes en la propia Filosofía de la ciencia. Muchos han pedido una mayor participación de filósofos de la ciencia en educación científica (Harms y Yagger, 1981; Koertge, 1969; Summers, 1972). Pero con algunas excepciones (como Israel Scheffler y Michael Martin) se han quedado sin respuesta. Joseph Agassi es otro. Su Historia de la física en forma de diálogo (entre él mismo y su hijo) es un ejemplo maravilloso de cómo un filósofo/historiador puede enriquecer la instrucción científica (Agassi, 1968).

La Filosofía potencia la enseñanza en clase. Una simple lección de mecánica se puede transformar si surgen preguntas sobre la relación de las teorías con los hechos, sobre qué se exige a un buen experimento, o por qué definimos por ejemplo aceleración como cambio de velocidad con respecto al tiempo más que a la distancia. Las clases de evolución son más atractivas si surgen preguntas sobre los límites adecuados de la ciencia y la religión o si se investigan preguntas sobre la realidad o conveccionalidad de las especies (un punto muy importante en la acogida del darwinismo). Puede introducirse a los niños en el aspecto metafísico del naturalismo de Darwin y sus ramificaciones para entender el lugar del hombre en la naturaleza. Pueden surgir cuestiones sobre cómo se creó la teoría de Darwin y la suficiencia de las pruebas en aquel tiempo. Se puede dirigir la atención a la aparente circularidad de la ley de supervivencia del mejor adaptado; o de las afirmaciones repetidas de gradualismo a pesar de tantas pruebas en su contra. En estos puntos pueden desarrollarse las perspectivas históricas y filosóficas, y pueden contribuir a una enseñanza mejor y más interesante de las ciencias.

Las clases de estructura atómica pueden dar la oportunidad de debatir modelos de la ciencia y el punto crucial de cómo se relacionan hechos y teoría, 
y de los fundamentos para elección de teorías. Los puntos básicos del realismo y del instrumentalismo pueden surgir de la manera adecuada. Los alumnos aprenden tradicionalmente el modelo «bola de billar», el modelo «pudding de ciruelas», el modelo «sistema solar». Este desfile tiene más interés y mordiente intelectual si se hacen algunas preguntas elementales sobre los modelos y el mundo. ¿Son retratos del mundo? ¿Son verdaderas las afirmaciones sobre ellos o sólo útiles? ¿Qué convierte una afirmación en verdadera? El famoso prefacio instrumentalista de Osiander a la obra de Copérnico Sobre las Revoluciones de las Esferas Celestes podría ser un punto de partida adecuado para tal discusión. Estas materias se han tratado en las obras sobre educación científica (Hodson, 1982; Lind, 1980; Ormell, 1980; Osborne y Gilbert, 1980).

La enseñanza de la combustión puede incluir una apreciación de la teoría del flogisto. Entendida históricamente, está claro que no era una teoría estúpida como a menudo se ha descrito. Puede preguntarse a los alumnos por sus puntos fuertes, hasta qué punto coincidía con la observación y por qué la teoría del flogisto fue defendida por científicos de los más reconocidos. Todos los asuntos que hacen surgir preguntas interesantes sobre el proceso de cambio científico y la evaluación de teorías. Una unidad de trabajo tan extensa tan sólo se ha probado en algunas escuelas inglesas. Este es el tipo de cooperación que se requiere entre historiadores y educacionalistas (Pumfrey, 1987).

Una simple lección sobre el péndulo puede potenciarse si se introducen puntos históricos sobre Galileo y sus disputas con sus oponentes aristotélicos. El punto crucial de si la ley del péndulo es «verdadera» sólo para péndulos «ideales», la importancia de frases como «siendo otras cosas iguales» en la formulación de leyes científicas y las conexiones entre descripciones matemáticas y a realidad física. Estos fueron puntos cruciales de la revolución científica. Se ilustran con la Historia de la física del movimiento pendular. Galileo decía de los péndulos pesados y ligeros (hierro y corcho) que, puestos en movimiento, permanecerán en sincronía «mil oscilaciones». Cuando los alumnos hacen el experimento los péndulos se desincronizan tras dos docenas de vaivenes. Algo que se confirma en reproducciones del experimento de Galileo (Naylor, 1984). ¿Qué nos dice esto sobre Galileo, sobre la relación de pruebas y teoría en la ciencia, sobre la interpretación de experimentos, sobre el lugar de la retórica en la ciencia? Y por encima de estos puntos está el fundamental de la matematización de la ciencia y la conexión entre fórmulas matemáticas y los hechos del mundo (Mattheus, 1987). No puede haber más que beneficio para los alumnos si se les expone estos puntos y se les pide que los mediten.

No hay materia que no pueda hacerse más interesante y atractiva con la introducción de consideraciones filosóficas, sean consideraciones sobre la visión del mundo, o de tipo técnico-analítico. El objeto no es enseñar Filosofía, sino potenciar el aprendizaje y favorecer una mayor consciencia de la exitación intelectual y los logros de la ciencia. Verla como una actividad cultural que afecta a otras áreas de la vida (religión, ética, Filosofía) y se ve a su vez afectada e influenciada por ellas. Empezar a comprender cómo y en qué sentido la ciencia nos da la mejor comprensión que tenemos del mundo en que vivimos.

Hay muchos campos fructíferos en la Filosofía de la ciencia relevantes para la educación científica. Cada vez se escriben más obras sobre la relación entre las chicas y las ciencias escolares, como puso en evidencia el número especial de 1987 del International Journal of Science Education (Diario Internacional de Educación Científica). Independientemente de esto, ha habido un vivo debate 
en la Filosofía de la ciencia sobre feminismo y epistemología, y sobre los supuestos machistas de la metodología y la Historia de la ciencia. Claramente la interrelación sólo puede beneficiar a ambas ramas.

La Filosofía de la Matemática es otro campo que puede aportar algo a la educación científica. Está claro que la ciencia moderna es Matemática. También está claro que una Matemática inadecuada es uno de los los mayores obstáculos para el desarrollo del conocimiento científico. Se ha trabajado en gran medida sobre la evolución histórica de la Matemática y los problemas filosóficos asociados. La contribución de Imre Lakatos es tal vez la más conocida (Lakatos, 1977). Los educadores de matemáticas han prestado atención a su obra y bajo la influencia del contructivismo la han aplicado a estudios de la psicología de la Matemática y en el desarrollo de programas y métodos de enseñanza (Glaserfeld, 1988; Sinclair, 1987). A pesar de que las matemáticas sean tan cruciales en la ciencia. Los científicos académicos han prestado poca atención a este curso de enseñanza de las matemáticas y Filosofía de la Matemática. Una vez más la colaboración sólo puede ser beneficiosa. Puede decirse lo mismo de la investigacion de la ciencia cognitiva.

El beneficio que obtienen los profesores de ciencias que tienen interés y competencia en Historia y Filosofía de la ciencia son obvios. Lo que se requiere es una mayor colaboración entre historiadores, filósofos y educadores científicos en la preparación de profesores, confección de material escolar, la dirección de la investigación y el análisis de libros de texto y programas. Esto ha sido así en alguna medida en el pasado. Hay signos esperanzadores de que será más corriente en el futuro.

\section{Referencias}

AGASSI, J. (1968). The continuing revolution: A bistory of physic from the Greeks to Einstein. Nueva York: McGraw Hill.

Aikenhead, G. S. (1974). Course evaluation II: Interpretation of student performance on evaluation tests. Joumal of Research in Science Teaching, II, 23-30.

ARChenhold, W., et al (Eds.). (1980). Cognitive development research in science E mathematics. Leeds: University of Leeds.

Armstrong. H. E. (1903). The teaching of scientific method. Londres: Macmillan.

Association For Science Education. (1979). Alternatives for science education, A consultative document. Hafield: Edición de autor.

Association For Science Education. (1980). What is science? Hatfield: Edición de autor.

Atkinson, P., \& Delamont, S. (1977). Mock-ups \& cock-ups. En M. Hammersley y P. Woods (Eds.) The Process of Schooling (pp. 87-108). Londres.

British Association For The Advancement Of Science. (1917). Report of the British Association for Advancement of Science. Londres: Murray.

Brock, W. H. (1987). History of science in British schools: Past, present \& future. Original no publicado, University of Leicester.

BRUmBY, M. (1979). Problems in learning the concept of natural selection. Joumal of biological Edu. cation, 13, 119-122.

Brusi, S. G. (1974). Should the history of science be rated X? Science, 18, 1164-1172.

Brusu, S. G. (1978). Why chemistry needs history and how it can get some. Journal of College Science Teaching, 7, 288-291.

Brush, S.G. (1987, Summer). History of science and science education. Scientific Literacy Papers, 75.85 .

BybEe, R. W. (1977). The new transformation of science education. Science Education, 61, (1), 85.97 .

BYBEE, R. W., et al (Eds.) (1984). Redesigning science E technology education. Washington DC: National Science Teachers Association. 
Chinapagne, A. B., Klopfer, L.E. y Anderson, J. (1980). Factors influencing learning of classical mechanics. American Joumal of pbysics, 48, 1074-1079.

Conant. (1964). Case studies in experimental science.

Crane L. T. (1976). The National Science Foundation \& pre-college science education: 1950-1975 Washington, DC: US Government Printing Office.

Department Of Education And Science. (1985). Science 5-16: A statement of policy. Londres. HMSO.

Disessa, A. A. (1982). Unlearning Aristotelian physics: A study of knowledge-based learning. Cognitive Science, $6,37-75$.

Dowling, K. y YAGER, R.E. (1983). Status of science education in state departments of education. Joumal of Research in Science Teaching, 20 (8), 771-780.

Driver, R., et al (Eds.) (1985). Children's ideas in science. Milton Keynes: Open University Press.

Driver, R., y Easley, J. (1978). Pupils \& paradigms: A reviex of literature related to concept development in adolescent science students. Studies in Science Education, 5, 61-84.

Duirm, P. (1954). The aim E structure of physical theory (P. P. Wiener, trans.). Princeton: Princeton University Press.

DusCHL, R. A. (1985). Science education \& philosophy of science, twenty-five years of mutually exclusive development. School Science \& Mathematics, 87 (7), 541-555.

Elbers, G. W. y Duncan, P. (Eds.) (1959). The scientific revolution: Challenge E promise. Washington DC: Public Affais Press.

EnNis, R. H. (1979). Research in philosophy of science bearing on science education. En P. D. Asquith y H. E. Kyburg (Eds.), Current research in philosopby of science. East Lansing: PSA.

Finocchinro, M. A. et al. (1980). A symposium on the use of the history of science in the science curriculum. Journal of College Science Teacbing, 10 (1), 14-33.

GAlLAGHER, J., y YAGER, R. E. (1981). Science educator perceptions of problems facing science education: A report of five surveys. Joumal of Research in Science Teaching 18 (6), 505-514.

Gilbert, J. K., Osborne, R. J., y Fensham, P. (1982). Childre's science \& its consequences for teaching. Science Education, 66 (4), 623.633.

Glasersfeld, E. von. (1987). Construction of knoweledge. Salinas, CA: Intersystems Publications

GLASERSFELD, E. von (Ed.) (1988). Constructivism in mathematics education. Dordrecht: Reidel.

HARMS, N. C., y Y $\Lambda$ GER, R. E. (1981). What research says to the science teacher (vol. 3). Washington DC: NSTA.

Helm, H., y Novak, J. D. (Eds.) (1983). Proceedings of the International Seminar on Misconceptions in Science $\&$ Mathematics. Ithaca: Education Department, Cornell University.

Hodson, D. (1982). The pursuit of truth? Parts I, II. School Science Review, 63, 225, 643-652; $226,23-30$ -

Honson, D. (1987). Social control as a factor in science curriculum change. International Joumal of Science Education, 9, 529-540.

Houron, G. (1978). On the educational philosophy of the Project Physics Course. In his The scientific imagination: Case studies. Cambridge: Cambridge University Press.

Holton, G. (1986). «A Nation At Risk» revisited. En The advancement of science $\mathcal{E}$ its burdens. Cambridge: Cambridge University Press.

Hurd, P. De H. (1984). Reforming science education: The search for a new vision. Washington DC: Council for Basic Education.

Kauffman, G. B. (1979). History in the chemistry curriculum: Pros y cons. Annals of Science, 36 , 395-402.

KLOPFER, L. (1969). The teaching of science \& the history of science. Joumal of Research in Science Teaching, 6, 87-95.

Koertge, N. (1969). Towarde an integration of content and method in science curriculum. Curriculum Theory Network, 4, 26-43

Kurn, T. S. (1970). The structure of scientific revolutions (2nd ed). Chicago: University of Chicago Press.

Kurin, T. S. (1977). Concepts of cause in the development of physics. Inhis The essential tension. Chicago: University of Chicago Press.

Lakntos, I. (1977). Proofs \& refutations. Cambridge: Cambridge University Press.

LIND, G. (1980). Models in physics: Some pedagogical reflections based on the history of science. European Joumal of Science Education, 2, 15-23.

LuCAs, A. M. (1971). Creativity, discovery, and inquiry in science education. Australian Joumal of Education, 15 (2), 185-196.

Maci, E. (1911). The history $\&$ root of the principle of the consevation of energy. Chicago: Open Court. (orig. 1872).

MAсн, E. (1895). On instruction in the classics \& the sciences. In Popular Scientific Lectures. Chicago: Open Court. (La Salle, 1943).

Martiews, M. R. (1987). Galileo's pendulum \& the objects of science. In B. y D. Arnstine (Eds.), Pbilosophy of Education. Philosophy aof education Society.

MCCloskey, M. (1983). Intuitive physics. Scientific American, 248, 114-122.

MANUEl, D. E. (1981). Reflections on the role of history \& philosophy of science in school scien ce education. School Science Review, 62 (221), 769-771. 
Martin, M. (1974). The relevance of philosophy of science for science education. Boston Studies in Pbilosopby of Science, 32, 293-300.

National Commission on Excellence in Education. (1983). A nation at risk: The imperative for educational reform. Washington DC: U. S. Department of Education.

National Science Board. (1983). Educating Americans for the 21 st century. Washington DC: Author.

NAYLoR, R. H. (1974). Galileo's simple pendulum. Physics, 16, 23-46.

Novak, J. D. (Ed.) (1987). Proceedings of the Second International Seminar on Misconceptions E Educational Strategies in Science E Mathematics. Ithaca: Education Department, Cornell University.

Omeron, M. D. (1971). The social implications factor in attitudes to science. British Joumal of Educational Psychology, 41, 335-338.

Ormell, E. (1980). Mathematical models \& understanding in science. The School Science Review, $62,219,375-381$.

Osborne, R. J., y Gilbert, J. K. (1980). The use of models in science teaching. School Science Review, 62, 218, 57-67.

Prundt, H., y Duit, R. (1988). Bibliography of students' alternative frameworks \& science education (2nd ed.). Intitute for Science Education, Universtity of Kiel.

Paget, J. (1970). Genetic epistemology. Londres: Columbia University Press.

Pumfrey, S. (1987). The concept of oxygen: Using history of science in science teaching. Original no publico, External Studies, Oxfork University.

Ramage, H. P. (1983). The treatment of the philosophy of science in the Science in Society Project. School Science Review, 64 (299), 786-787.

RoBerts, D. A. y Russell T. (1975). An alternative approach to science education research: Drawing from philosophical analysis to examine practice. Curriculum Theory Network, 5, 107-125.

Robinson, J. T. (1969). Philosophy of science: Implications for teacher education. Joumal of Research in Science Teaching, 6, 99-104.

RusSELL, T. L. (1981). What history of science, how much and why? Science Education, 65, 51-64.

Schlipp, P.A. (1951). Albert Einstein (2nd ed.). Nueva York: Tudor.

ScriwaB, J. J. (1962). The teaching of science as enquiry. En J. J. Schwab y P. Brandwein (Eds.), The taching of science. Cambridge: Harvard University Press.

Sierratt, W. J. (1982). History of science in the science curriculum: an historical perspective. Scbool Science Review, 64, 225-236, 418-424.

Sinclarr, N. (1987). Constructivism \& the psychology of mathematics. En N. Herscovics y C. Kieran (Eds.), Proceedings of the Eleventh Conference of the International Group for the Psychology of Mathematics Education. Universidad de Montreal, Montreal.

Summers, M. K. (1982). Philosophy of science in the science teacher education curriculum. European Journal of Science Education, 4, 19-28.

Thompson, J. J. (1987). National curriculum interim report: Science. Londres: DES.

VIENNOT, L. (1979). Spontaneous reasoning in elementary dynamics. European Joumal of Science Education, 1, 205-221.

W ARING, M. (1979). Social pressures E curriculum innovation: a study of the Nuffield Foundation Science Teaching Project. Londres: Methuen.

WEISS, I. T. (1978). Report of the 1977 National Survey of Science, Mathematics E Social Studies Education. Washington DC: U. S. Government Printing Office.

Welch, W. (1979). Twenty years of science education development: A look back. Review of Research in Education, 7, 282-306.

WELCII, W. ET AL (1981). The role of inquiry in science education: Analysis y recommendations. Science Education, 65, 33-50.

W HITAKER, R. J. (1983). Aristotle is not dead: Student understanding of trajectory motion. American Journal of Pbysics, 51 (4), 352-357.

Yager R. E. y Penick, J. E. (1985). Taking New Goals for School Science Seriously. Educational Leadership, $42(8), 86-87$. 